\title{
Use of the bond-projected superposition principle in determining the conditional probabilities of orbital events in molecular fragments
}

\author{
Roman F. Nalewajski
}

Received: 7 May 2010 / Accepted: 15 October 2010 / Published online: 4 November 2010

C The Author(s) 2010. This article is published with open access at Springerlink.com

\begin{abstract}
The problem of defining and determining the multi-conditional probabilities of many-orbital events in the chemical bond system of a molecule is addressed anew within theoretical framework of the one-determinantal orbital representation of molecular electronic structure. Its solution is vital for determining the information-theoretic indices of bond couplings between molecular fragments or the reactant/product subsystems in chemical reactions. The superposition principle of quantum mechanics, appropriately projected into the occupied subspace of molecular orbitals, is used to condition the atomic orbitals or general basis functions of the self-consistent-field calculations. The conditional probabilities between the subspaces of basis functions (atomic orbitals) are derived from an appropriate generalization of the bond-projected superposition principle. They are then used to define the triply-conditional probabilities, relating one conditional event to another. The resulting expression is shown to satisfy the relevant non-negativity and symmetry requirements. It is applied to probe the $\pi$-bond coupling in butadiene and benzene.
\end{abstract}

Keywords Bond orders - Bond projections - Conditional probabilities of orbitals · Couplings of chemical bonds · Density matrix · Information theory ·

Many-orbital events · Molecular fragments · Molecular information channels ·

Orbital communication theory $\cdot$ Superposition principle for subspaces

Throughout the paper $A$ denotes a scalar quantity, $\boldsymbol{A}$ stands for a row-vector, and A represents a square or rectangular matrix.

R. F. Nalewajski (凶)

Depatment of Theoretical Chemistry, Jagiellonian University, R. Ingardena 3, 30-060 Cracow, Poland e-mail: nalewajs@chemia.uj.edu.pl 


\section{Introduction}

In the Orbital Communication Theory (OCT) of the chemical bond [1-5], which explores the information propagation in the orbitally-resolved molecular communication channels [6-9], the overall information-theoretic (IT) bond orders and their entropic covalent (communication "noise") and ionic (information "flow") components are generated from the appropriate conditional probabilities between Atomic Orbitals (AO) contributed by the system constituent atoms, generated by the bondprojected superposition principle of quantum mechanics $[1-5,10]$. The basis functions (AO) of the standard Self-Consistent Field (SCF) calculations determine a natural set of the elementary electron-occupation events within this orbital approximation of the molecular electronic structure. The ultimate goal of these orbital (Hartree-Fock or Kohn-Sham) theories is to determine the subspace of the occupied Molecular Orbitals (MO), which determine the system chemical bonds and the equilibrium (ground-state) distribution of electrons in molecules.

Recently, the Communication Theory of the Chemical Bond (CTCB) $[1,6]$ has been extended to cover many-orbital events in the bond system of the molecule [1,3-5]. This development allows one to treat diverse bond-coupling phenomena between molecular fragments and reactant subsystems, which are often discussed in chemistry. For example, the alternative measures of the joint/conditional "probabilities" of the simultaneous two-orbital probability scatterings on different sites can be used to determine the IT-covalent and IT-ionic indices describing the mutual influences in the multi-site reactivity processes and catalysis, and it is capable of describing the coupling between the inter-reactant/product bonds in the concerted bond-forming/bond-weakening processes.

However, this promising many-orbital OCT perspective still suffers from several non-uniquenes and symmetry problems [1,3-5]. The previously designed, preliminary schemes within the superposition-principle approach, which have been used in the past to define the joint probabilities in the molecular bond system involving several AO, and the associated multi-conditional probabilities required to relate probability scatterings in different molecular fragments, still suffer from several shortcomings, e.g., non-positiveness and violation of some symmetries with respect to orbital exchanges. This hampers a wider use of the entropy/information descriptors they generate in interpreting subtle phenomena of the bond interactions in chemistry. It is the main purpose of this work to remedy this problem. The generalized superposition principle [1] for subspaces of basis functions, which closely follows the successful two-orbital development [2], will be shown to express the triply-conditional probabilities reflecting dependencies between information channels of molecular fragments in terms of elements of the molecular first-order density matrix.

\section{Two-orbital probabilities in OCT}

We begin with a short overview of the molecular communication systems in the $\mathrm{AO} /$ basis-function resolution and propagation of the condensed electron probabilities of $\mathrm{AO}$ in the molecular bond system [1,2]. The underlying conditional probabilities 
generate the entropy/information descriptors of the pattern of chemical bonds and their covalent and ionic composition in the molecular system under consideration. The conditional-entropy (communication noise) and mutual-information (information flow) descriptors of the molecular channel then provide the IT measures of the system covalency and ionicity, respectively $[1,6]$.

Consider the standard Restricted Hartree-Fock (RHF) description of the molecular electronic structure, in which a network of chemical bonds between constituent atoms is determined by the occupied Molecular Orbitals (MO) $\boldsymbol{\varphi}=\left\{\varphi_{s}\right\}$ in the system ground-state. Assuming, for reasons of simplicity, the closed-shell configuration of $N=2 n$ electrons then identifies the $n$ lowest (doubly-occupied, orthonormal) MO, $\boldsymbol{\varphi}=\left(\varphi_{1}, \varphi_{2}, \ldots, \varphi_{n}\right)$, as the origins of all bonds in the molecular system under consideration, both global (in the system as a whole) and regional (in molecular fragments). In the familiar LCAO MO approach MO are represented by the linear combinations $\boldsymbol{\varphi}=\chi \mathbf{C}$ of the adopted orthonormal basis functions, $\chi=\left(\chi_{1}, \chi_{2}, \ldots, \chi_{m}\right)=$ $\left\{\chi_{i}\right\},\langle\chi \mid \chi\rangle=\left\{\delta_{i, j}\right\} \equiv \mathbf{I}$, e.g., Löwdin's symmetrically orthogonalized AO; here, the rectangular matrix $\mathbf{C}=\left\{C_{i, s}\right\}=\langle\chi \mid \varphi\rangle$ groups the LCAO MO expansion coefficients, to be determined from the energy variational principle using the familiar Self-Consistent Field (SCF) procedure. The occupied MO thus determine the modes of the information propagation in the molecule, due to electron delocalization via the system chemical bonds.

The basis functions $\chi$ define the underlying elementary "events" determining the channel inputs $\boldsymbol{a}=\left\{\chi_{i}\right\}$ and outputs $\boldsymbol{b}=\left\{\chi_{j}\right\}$, with the associated probabilities of finding an electron on these functions $\boldsymbol{P}(\boldsymbol{a})=\left\{P\left(\chi_{i}\right)=p_{i}\right\}=\boldsymbol{p}$ and $\boldsymbol{P}(\boldsymbol{b})=\left\{P\left(\chi_{j}\right)=\right.$ $\left.q_{j}\right\}=\boldsymbol{q}$. The information network describing the orbital communications in the molecule is determined by the conditional probabilities of the channel outputs given inputs, $\mathbf{P}(\boldsymbol{b} \mid \boldsymbol{a})=\left\{P\left(\chi_{j} \mid \chi_{i}\right)=P(j \mid i)\right\}, \sum_{j} P(j \mid i)=1$. They describe the probability propagation via the system chemical bonds and are characterized by standard quantities developed in IT for real communication devices. The electron-delocalization in molecules via the system chemical bonds is embodied in the probability spread of each row $\{\boldsymbol{P}(\boldsymbol{b} \mid i)\}$ of $\mathbf{P}(\boldsymbol{b} \mid \boldsymbol{a})$. In OCT these conditional probabilities follow from the quantummechanical superposition principle [10] supplemented by the "physical" projection of $\mathrm{AO}$ onto the subspace of the system occupied MO [1-5].

To summarize, the molecular information network in the AO resolution involves the full list of the AO-events $\chi$ in its "input" $\boldsymbol{a}=\left\{\chi_{i}\right\}$ and "output" $\boldsymbol{b}=\left\{\chi_{j}\right\}$. It represents the effective communication promotion of these basis functions in the molecule, via the probability/information scattering described by the conditional probabilities of the AO-outputs given AO-inputs in the molecular bond system, identified by the row (input) and column (output) indices $i$ and $j$, respectively. In OCT the entropy/information indicators of the covalent and ionic components of all chemical bonds in the given molecular system represent the complementary descriptors of the average communication-noise and information-flow in this molecular information channel.

In this orbital description the $\mathrm{AO} \rightarrow \mathrm{AO}$ "communications" are thus fully characterized by the conditional probabilities $\mathbf{P}(\boldsymbol{b} \mid \boldsymbol{a})=\{P(i \wedge j) / P(i)\}$ of the output AO-events $\{\boldsymbol{b}\}$, given the input AO-events $(\boldsymbol{a})$, where the associated joint probabilities of simultaneously observing two AO in the system chemical bonds, $\mathbf{P}(\boldsymbol{a} \wedge \boldsymbol{b})=\{P(i \wedge j)\}$, 
satisfy the usual normalizations:

$$
\sum_{i} P(i \wedge j)=P(j), \quad \sum_{j} P(i \wedge j)=P(i), \quad \sum_{i} \sum_{j} P(i \wedge j)=1 .
$$

The key quantity of the standard one-determinantal description is the first-order density matrix $\boldsymbol{\gamma}$ in the AO representation, also called the Charge-and-Bond-Order (CBO) matrix. It represents the projection operator onto the subspace of all (doubly occupied) MO, $\hat{\mathrm{P}}_{\varphi}=|\varphi\rangle\left\langle\varphi\left|=\sum_{s}\right| \varphi_{s}\right\rangle\left\langle\varphi_{s}\right| \equiv \sum_{s} \hat{\mathrm{P}}_{s}, \hat{\mathrm{P}}_{\varphi}^{2}=\hat{\mathrm{P}}_{\varphi}$,

$$
\begin{aligned}
\boldsymbol{\gamma} & =2\langle\chi \mid \varphi\rangle\langle\varphi \mid \chi\rangle=2 \mathbf{C C}^{\dagger} \equiv 2\left\langle\chi\left|\hat{\mathrm{P}}_{\varphi}\right| \chi\right\rangle \\
& =\left\{\gamma_{i, j}=2\left\langle\chi_{i}\left|\hat{\mathrm{P}}_{\varphi}\right| \chi_{j}\right\rangle \equiv 2\left\langle i\left|\hat{\mathrm{P}}_{\varphi}\right| j\right\rangle \equiv 2\left\langle i^{b} \mid j^{b}\right\rangle\right\}
\end{aligned}
$$

where $\hat{\mathrm{P}}_{\varphi}|i\rangle=\left|i^{b}\right\rangle=|\varphi\rangle\langle\varphi \mid i\rangle$ stands for the projection of $\chi_{i}$ onto the bond-subspace $\boldsymbol{\varphi}$, satisfying the associated closed-shell idempotency relation:

$$
(\gamma)^{2}=4\left\langle\chi\left|\hat{\mathrm{P}}_{\varphi}\right| \chi\right\rangle\left\langle\chi\left|\hat{\mathrm{P}}_{\varphi}\right| \chi\right\rangle=4\left\langle\chi\left|\hat{\mathrm{P}}_{\varphi}^{2}\right| \chi\right\rangle=4\left\langle\chi\left|\hat{\mathrm{P}}_{\varphi}\right| \chi\right\rangle=2 \gamma
$$

The CBO matrix reflects the promoted (valence) state of $\mathrm{AO}$ in the molecule. Its diagonal elements combine the effective electron occupations of the basis functions in the molecular ground-state, $\left\{N_{i}=\gamma_{i, i}=N p_{i}\right\}$, where $\boldsymbol{p}=\left\{p_{i} \equiv P\left(\chi_{i}\right)=\gamma_{i, i} / N\right\}$ stand for the (normalized) probabilities of AO being occupied in the molecule: $\sum_{i} p_{i}=N^{-1} \operatorname{Tr} \gamma=1$. This matrix also determines the system electron density,

$$
\rho(\boldsymbol{r})=2 \boldsymbol{\varphi}(\boldsymbol{r}) \boldsymbol{\varphi}^{\dagger}(\boldsymbol{r})=\chi(\boldsymbol{r})\left[2 \mathbf{C} \mathbf{C}^{\dagger}\right] \chi^{\dagger}(\boldsymbol{r}) \equiv \chi(\boldsymbol{r}) \boldsymbol{\gamma} \chi^{\dagger}(\boldsymbol{r})=N p(\boldsymbol{r}),
$$

and the one-electron probability distribution $p(\boldsymbol{r})=\rho(\boldsymbol{r}) / N$, the shape factor of $\rho$.

In quantum mechanics the geometric $(g)$ conditional probability $P^{g}(\phi \mid \psi)$ of observing a normalized (variable) state, say $\phi$, given another (parameter, reference) state, say $\psi$, emerges in the context of the superposition principle [10]:

$$
P^{g}(\phi \mid \psi)=|\langle\psi \mid \phi\rangle|^{2}=\langle\phi \mid \psi\rangle\langle\psi \mid \phi\rangle=\left\langle\phi\left|\hat{\mathrm{P}}_{\psi}\right| \phi\right\rangle=\left\langle\psi\left|\hat{\mathrm{P}}_{\phi}\right| \psi\right\rangle=P^{g}(\psi \mid \phi) .
$$

It should be observed that the quantum state conditioned upon itself in the molecular Hilbert space gives the probability of the sure event: $P^{g}(\psi \mid \psi)=1$.

Since we are interested in the simultaneous AO events occurring in the bond system of the molecule the two scalar products in the preceding equations have to be calculated between the AO projections into the occupied subspace $\varphi$ of MO [1,2]. Such physical conditional probabilities between $\mathrm{AO}$ are obtained by inserting the projector $\hat{\mathrm{P}}_{\varphi}$ between the two states, say $\phi=\chi_{j}$ and $\psi=\chi_{i}$, involved in the two scalar products of the preceding geometrical expression:

$$
\begin{aligned}
& P(j \mid i)=\mathcal{W}_{i}\left|\left\langle i\left|\hat{\mathrm{P}}_{\varphi}\right| j\right\rangle\right|^{2}=\mathcal{\alpha}_{i}\left\langle j\left|\hat{\mathrm{P}}_{\varphi}\right| i\right\rangle\left\langle i\left|\hat{\mathrm{P}}_{\varphi}\right| j\right\rangle=\mathcal{W}_{i}\left\langle j\left|\hat{\mathrm{P}}_{\varphi} \hat{\mathrm{P}}_{i} \hat{\mathrm{P}}_{\varphi}\right| j\right\rangle \\
& \equiv \gamma_{i}\left\langle j\left|\hat{\mathrm{S}}_{i}\right| j\right\rangle=\left(2 \gamma_{i, i}\right)^{-1} \gamma_{i, j} \gamma_{j, i}
\end{aligned}
$$


This probability in thus determined as expectation value in the final state $\chi_{j}$ of the molecular scattering operator $\hat{\mathrm{S}}_{i}$ from the initial (reference) state $\chi_{i}$ :

$$
\hat{\mathrm{S}}_{i}=\hat{\mathrm{P}}_{\varphi}|i\rangle\left\langle i\left|\hat{\mathrm{P}}_{\varphi}=\hat{\mathrm{P}}_{\varphi} \hat{\mathrm{P}}_{i} \hat{\mathrm{P}}_{\varphi}=\right| i^{b}\right\rangle\left\langle i^{b}\right| .
$$

The proportionality constant $\mathcal{\gamma}_{i}=\left(2 \gamma_{i, i}\right)^{-1}$ satisfies the required normalization condition [see Eq. 3]:

$$
\sum_{j} P(j \mid i)=\gamma_{i} \sum_{j} \gamma_{i, j} \gamma_{j, i}=2 \gamma_{i} \gamma_{i, i}=1
$$

Due to its dependence on the occupation $\gamma_{i, i}$ of the reference state $\chi_{i}$ in the molecular bond system the symmetry of Eq. 5 is observed only for equall effective occupations of both $\mathrm{AO}$ in the molecule.

One should also observe that for the identical AO in the conditioned pair,

$$
P(i \mid i)=\gamma_{i, i} / 2=1 / 2 N p_{i}
$$

This diagonal probability thus measures the number of electronic pairs on $\chi_{i}$ and reflects the probability $p_{i}$ of this $\mathrm{AO}$ event in the molecule. This result correctly identifies the sure event of detecting electron on $\chi_{i}$ only for the double (full) occupation of this AO in the molecule, when $N_{i}=\gamma_{i, i}=2$. This full orbital occupation also signifies that this AO does not participate in any chemical interactions with other orbitals, thus remaining purely non-bonded in character.

The off-diagonal conditional probability of $j$ th AO-output given $i$ th AO-input is thus proportional to the square of the $\mathrm{CBO}$ matrix element linking the two $\mathrm{AO}$, which represents [see Eq. 2] the scalar product of the bond projections of two AO onto the bond subspace $\varphi$. Therefore, this two-AO probability is also proportional to the corresponding contribution $\mathrm{C} \kappa_{i, j}=\gamma_{i, j}^{2}$ to the Wiberg [11] index of the chemical bond-order between the constituent atoms $\mathrm{A}$ and $\mathrm{B}$ in the molecule,

$$
\operatorname{dr}(\mathrm{A}, \mathrm{B})=\sum_{i \in \mathrm{A}} \sum_{j \in \mathrm{B}} d \kappa_{i, j}
$$

and to related quadratic descriptors of the molecular bond-multiplicities [12-21].

Using Eq. 3 it can be now readily verified that the associated matrix of the joint AO probabilities,

$$
\mathbf{P}(\boldsymbol{a} \wedge \boldsymbol{b})=\left\{P(i \wedge j)=P(i) P(j \mid i)=(2 N)^{-1} \gamma_{i, j} \gamma_{j, i}\right\},
$$

satisfies the normalization conditions of Eq. 1, e.g.,

$$
\sum_{i} P(i \wedge j)=(2 N)^{-1} \sum_{i} \gamma_{j, i} \gamma_{i, j}=(2 N)^{-1} 2 \gamma_{j, j}=P(j) .
$$


These relations also imply that molecular input probabilities $\boldsymbol{P}(\boldsymbol{a}) \equiv \boldsymbol{p}$ generate the same probabilities in the output of the molecular channel,

$$
\boldsymbol{P}(\boldsymbol{b})=\boldsymbol{p} \mathbf{P}(\boldsymbol{b} \mid \boldsymbol{a})=\left\{\sum_{i} P(i) P(j \mid i)=\sum_{i} P(i \wedge j)\right\}=\boldsymbol{p},
$$

thus confirming the stationary character of this distribution.

The conditional probabilities of Eq. 6 explore the dependencies between AO resulting from their simultaneous participation in the system occupied MO, i.e., their involvement in the entire network of chemical bonds in the molecule. They define the probability scattering in the AO-channel of the molecule, in which the "signals" of the molecular/promolecular electron allocations to basis functions are transmitted from the AO inputs to AO outputs. Such information system constitutes the basis of OCT of the chemical bond [1-5]. This molecular channel can be probed using both the promolecular $\left(\boldsymbol{p}^{0}=\left\{p_{i}^{0}\right\}\right)$ and molecular $(\boldsymbol{p})$ input probabilities, in order to extract the IT-multiplicities of the ionic and covalent bond components, respectively. The atomic promolecule is defined by a collection of the "frozen" constituent (free) atoms brought to their final (molecular) locations. The AO probabilities $\boldsymbol{p}^{0}$ thus reflect the corresponding ground-states of the system separated atoms, which specify the initial state in the bond-formation process, in the spirit of the familiar density-difference function:

$$
\Delta \rho(\boldsymbol{r})=\rho(\boldsymbol{r})-\rho^{0}(\boldsymbol{r})=N\left[p(\boldsymbol{r})-p^{0}(\boldsymbol{r})\right],
$$

where $\rho^{0}(\boldsymbol{r})$ and $p^{0}(\boldsymbol{r})=\rho^{0}(\boldsymbol{r}) / N$ stand for the promolecular electron density and its shape/probability factor, respectively.

It should be emphasized that the elementary geometrical events $\{i\}=|\chi\rangle$, of finding an electron on the specified basis functions $\left\{\chi_{i}\right\}$, e.g., the orthogonalized AO, correspond to the pure quantum states $\{|i\rangle\}$ identified by their individual projection operators $\left\{\hat{\mathrm{P}}_{i}=|i\rangle\langle i|\right\}$, with the whole basis-function space corresponding to the overall projection $\hat{\mathrm{P}}_{\chi}=|\chi\rangle\langle\chi|=\sum_{i} \hat{\mathrm{P}}_{i}$. The physical events are conditional on the molecular ground state $|\psi\rangle$, approximated by a single Slater determinant defined by $|\boldsymbol{\varphi}\rangle=|\chi\rangle$ C. Therefore, as indeed reflected by the MO-projected expression of Eq. 6, the $\mathrm{AO}$ events in the molecular bond system refer to the mixed-state reflected by the density operator:

$$
\hat{\mathrm{D}}_{\chi}=\frac{1}{2}|\chi\rangle \boldsymbol{\gamma}\left\langle\chi\left|=\frac{1}{2} \sum_{i} \sum_{j}\right| i\right\rangle \gamma_{i, j}\langle j|=\sum_{i} \sum_{j} \hat{\mathrm{P}}_{i} \hat{\mathrm{P}}_{\varphi} \hat{\mathrm{P}}_{j} .
$$

This general observation, that elementary events are associated with the pure quantum states, i.e., with a single state vector in the molecular Hilbert space, while the conditional events correspond to the mixed quantum states, i.e., the ensembles defined by the relevant density operators, is essential for the multi-conditional development of Sect. 4, where we shall attempt to relate one conditional event upon another. Such 
probabilities should be thus associated with mixtures of the ensembles corresponding to the two conditional events in molecular subsystems.

\section{Conditioning of orbital subspaces}

It follows from Eq. 5 that the essence of the quantum conditioning of two states in $P^{g}(\phi \mid \psi)$, the expectation value in the variable state of the projector onto the parameter state, is the projection operator onto the reference state. This prescription is then supplemented in Eq. 6 by the additional projection onto the occupied subspace of MO, which reflects the actual participation of $\mathrm{AO}$ involved in the molecular chemical bond system. It is the main goal of this section to devise a similar procedure for conditioning the AO subspaces. In the next section we shall use this generalization to condition the two-AO scattering events on different molecular subsystems.

The same bond-projected superposition principle will now be used to derive these singly-conditional probabilities, which simultaneously involve larger subspaces of basis functions. Let us specifically examine the quantum conditioning of two subspaces in $\chi$, spanned by the specified pairs of AO, each containing two basis functions: $\varepsilon_{1}=\left\{\chi_{i}, \chi_{j}\right\} \equiv\{u(1)\}$ and $\varepsilon_{2}=\left\{\chi_{k}, \chi_{l}\right\} \equiv\{w(2)\}$. These orbital pairs define the associated subspace projectors,

$$
\hat{\mathrm{P}}_{\varepsilon_{1}}=|i\rangle\langle i|+| j\rangle\left\langle j\left|=\hat{\mathrm{P}}_{i}+\hat{\mathrm{P}}_{j}, \quad \hat{\mathrm{P}}_{\varepsilon_{2}}=\right| k\right\rangle\langle k|+| l\rangle\langle l|=\hat{\mathrm{P}}_{k}+\hat{\mathrm{P}}_{l},
$$

A natural generalization of the procedure adopted in the two-AO development of Eq. 6 calls for the trace over the variable subspace $\varepsilon_{1}$ of the projector onto the reference subspace $\varepsilon_{2}$. This gives the following geometric $(g)$ expression, corresponding to the whole molecular Hilbert space,

$$
\begin{aligned}
& P^{g}\left(\varepsilon_{1} \mid \varepsilon_{2}\right)=\mathcal{\nu \gamma}^{g} \sum_{u(1)}\left\langle u(1)\left|\hat{\mathrm{P}}_{\varepsilon_{2}}\right| u(1)\right\rangle \\
& =\mathcal{d r} \sum_{w(2)}\left\langle w(2)\left|\hat{\mathrm{P}}_{\varepsilon_{1}}\right| w(2)\right\rangle=P^{g}\left(\varepsilon_{2} \mid \varepsilon_{1}\right) \\
& =\left\langle\gamma^{g}[\langle i \mid k\rangle\langle k \mid i\rangle+\langle i \mid l\rangle\langle l \mid i\rangle+\langle j \mid k\rangle\langle k \mid j\rangle+\langle j \mid l\rangle\langle l \mid j\rangle]\right. \\
& =\mathcal{\alpha r}^{g}\left(\delta_{i, k}+\delta_{i, l}+\delta_{j, k}+\delta_{j, l}\right) \text {, }
\end{aligned}
$$

where $\delta^{g}$ denotes the appropriate normalization constant. Its physical, bond-projected analog then reads:

$$
\begin{aligned}
P\left(\varepsilon_{1} \mid \varepsilon_{2}\right) & =\gamma_{2} \sum_{u(1)}\left\langle u(1)\left|\hat{\mathrm{P}}_{\varphi} \hat{\mathrm{P}}_{\varepsilon_{2}} \hat{\mathrm{P}}_{\varphi}\right| u(1)\right\rangle \equiv \gamma_{2} \operatorname{Tr}_{\varepsilon_{1}} \hat{\mathrm{S}}_{\varepsilon_{2}} \\
& =\gamma_{2}\left(\gamma_{i, k} \gamma_{k, i}+\gamma_{i, l} \gamma_{l, i}+\gamma_{j, k} \gamma_{k, j}+\gamma_{j, l} \gamma_{l, j}\right) \\
& =\gamma_{2}\left[\left(\gamma_{i, k}\right)^{2}+\left(\gamma_{i, l}\right)^{2}+\left(\gamma_{j, k}\right)^{2}+\left(\gamma_{j, l}\right)^{2}\right] \geq 0,
\end{aligned}
$$


where the probability scattering operator from the reference subspace [compare Eq. 7]

$$
\hat{\mathrm{S}}_{\varepsilon_{2}}=\hat{\mathrm{P}}_{\varphi} \hat{\mathrm{P}}_{\varepsilon_{2}} \hat{\mathrm{P}}_{\varphi} \equiv \hat{\mathrm{P}}_{\varepsilon_{2}^{b}}=\sum_{w \in \varepsilon_{2}}\left|w^{b}\right\rangle\left\langle w^{b}\right| .
$$

As required, the resulting expression for the non-negative conditional probability $P\left(\varepsilon_{1} \mid \varepsilon_{2}\right)$ is seen to be symmetrical with respect to exchanges of orbitals inside and between the two subspaces. It combines the (renormalized) additive $t w o$-orbital contribution for all selections of single orbitals in the variable and parameter subspaces, respectively. It is straightforward to calculate once the self-consistent density matrix is known. This result can be extended to cover any number of basis functions in each subspace. Such conditional probabilities reflect communications between members of the two spaces involved and generate the associated IT covalent and ionic descriptors of the collective chemical interactions between these groups of orbitals in the molecular ground-state.

Again, the proportionality constant $\gamma_{2}$ has to satisfy the relevant normalization condition, the sum rule over all admissible selections of AO-pairs in the dependent set $\varepsilon_{1}$ :

$$
\operatorname{tr}_{\{\varepsilon(i, j)\}} P(i, j \mid k, l)=1 ;
$$

here $\operatorname{tr}_{\{\varepsilon(i, j)\}}=\operatorname{tr}_{\cup\{(i, j)\}}=\operatorname{tr}_{\varphi}$ denotes the trace over the union of all double-AO subspaces in the molecular bond space spanned by the orthogonal MO $|\varphi\rangle$, for all selections of both AO indices. The resulting subspace exhausts the whole bond space itself, determined also by the non-orthogonal bond-projections $\left|\chi^{b}\right\rangle$ of all basis functions. Indeed, since only electrons carry the physical information in the molecule, the preceding equation expresses the fact that the signal originating from the subspace $\varepsilon_{2}=$ $\left\{\chi_{k}, \chi_{l}\right\}$ will be totally scattered inside the bonding space of the doubly occupied MO,

$$
\sum_{\varepsilon_{1}} \hat{\mathrm{P}}_{\varepsilon_{1}^{b}}=\hat{\mathrm{P}}_{\varphi}=\hat{\mathrm{P}}_{\chi^{b}}=\sum_{i}\left|i^{b}\right\rangle\left\langle i^{b}\right| .
$$

Therefore, the overall normalization is expressed by the trace over the whole bonding space, which amounts to a single sumation over the repeated index of products of CBO matrix elements of any of the basis functions of $\varepsilon(i, j)$ in Eq. 17:

$$
\begin{aligned}
\operatorname{tr}_{\{\varepsilon(i, j)\}}\left(\gamma_{k, i} \gamma_{i, k}+\gamma_{i, l} \gamma_{l, i}+\gamma_{j, k} \gamma_{k, j}+\gamma_{j, l} \gamma_{l, j}\right) \equiv & \sum_{i}\left(\gamma_{i, k} \gamma_{k, i}+\gamma_{l, i} \gamma_{i, l}\right) \\
& +\sum_{j}\left(\gamma_{k, j} \gamma_{j, k}+\gamma_{l, j} \gamma_{j, l}\right) \\
& =4\left(\gamma_{k, k}+\gamma_{l, l}\right) .
\end{aligned}
$$

Finally, the normalization condition of Eq. 19 reads:

$$
\gamma_{2} \operatorname{tr}_{\varphi} \hat{\mathrm{S}}_{\varepsilon_{2}}=\alpha_{2} 4\left(\gamma_{k, k}+\gamma_{l, l}\right)=1 \text {, }
$$

and hence $\gamma_{2}=1 /\left[4\left(\gamma_{k, k}+\gamma_{l, l}\right)\right]$. 
The final expression for the singly-conditional probability relating in the bond space one two-AO subspace to another two-AO subspace,

$$
P(i, j \mid k, l)=\left[4\left(\gamma_{k, k}+\gamma_{l, l}\right)\right]^{-1}\left(\gamma_{i, k} \gamma_{k, i}+\gamma_{i, l} \gamma_{l, i}+\gamma_{j, k} \gamma_{k, j}+\gamma_{j, l} \gamma_{l, j}\right),
$$

constitutes a natural generalization of the two-AO expression of Eq. 6. As expected this probability vanishes when there is no chemical coupling between the two AO-subsets, $\gamma_{i, k}=\gamma_{i, l}=\gamma_{j, k}=\gamma_{j, l}=0$, either because of the symmetry requirements or due to their large spatial separation $R_{1,2} \rightarrow \infty$ :

$$
P(i, j \mid k, l) \rightarrow 0\left(R_{1,2} \rightarrow \infty\right)
$$

This probability is seen to assume a general form of the known two-orbital expression of Eq. 6,

$$
P\left(\varepsilon_{1} \mid \varepsilon_{2}\right)=\gamma_{\varepsilon_{1}, \varepsilon_{2}}^{2} /\left(2 \gamma_{\varepsilon_{2}, \varepsilon_{2}}\right)
$$

when expressed in terms of the average "chemical" coupling $\gamma_{\varepsilon_{1}, \varepsilon_{2}}$ between the two conditioned subspaces,

$$
\gamma_{\varepsilon_{1}, \varepsilon_{2}}^{2}=1 / 4\left[\left(\gamma_{i, k}\right)^{2}+\left(\gamma_{i, l}\right)^{2}+\left(\gamma_{j, k}\right)^{2}+\left(\gamma_{j, l}\right)^{2}\right]
$$

and the average $\mathrm{AO}$ electron occupation in the parameter subspace,

$$
\gamma_{\varepsilon_{2}, \varepsilon_{2}}=\frac{1}{2}\left(\gamma_{k, k}+\gamma_{l, l}\right)
$$

This conditional probability also defines the associated joint-probability of the two subspaces in the bonding space of the molecule:

$$
\begin{aligned}
P[(i, j) \wedge(k, l)] \equiv & P(k \wedge l) P(i, j \mid k, l) \\
= & {\left[8 N\left(\gamma_{k, k}+\gamma_{l, l}\right)\right]^{-1}\left(\gamma_{k, l}\right)^{2}\left[\left(\gamma_{i, k}\right)^{2}+\left(\gamma_{i, l}\right)^{2}\right.} \\
& \left.+\left(\gamma_{j, k}\right)^{2}+\left(\gamma_{j, l}\right)^{2}\right],
\end{aligned}
$$

which also conforms to the adopted normalization over the union of all admissible two-AO subspaces:

$$
\begin{aligned}
\operatorname{tr}_{\{\varepsilon(i, j)\}} P[(i, j) \wedge(k, l)] & =\left[8 N\left(\gamma_{k, k}+\gamma_{l, l}\right)\right]^{-1}\left(\gamma_{k, l}\right)^{2} 4\left(\gamma_{k, k}+\gamma_{l, l}\right) \\
& =\left(\gamma_{k, l}\right)^{2} /(2 N)=P(k \wedge l) \equiv P(k, l) .
\end{aligned}
$$




\section{Conditioning of probability propagations in molecular fragments}

Let us now address the associated problem of conditioning the probability propagations in molecular fragments. In communication theory the localized chemical interaction between the given pair $\left\{\chi_{i}, \chi_{j}\right\}$ of AO reflects the probability scattering between these two basis functions. It is embodied in the respective elements $P(j \mid i)$ and $P(i \mid j)$ of the conditional probability matrix $\mathbf{P}(\boldsymbol{b} \mid \boldsymbol{a})$. In order to quantify in IT the coupling effects between such localized chemical bonds, say between $\chi_{i}$ and $\chi_{j}$ in one molecular fragment, and between $\chi_{k}$ and $\chi_{l}$ in another part of the molecule, when $\varepsilon_{1}=\left(\chi_{i}, \chi_{j}\right)$ and $\varepsilon_{2}=\left(\chi_{k}, \chi_{l}\right)$ are disjoint, one has to relate the two conditional events $\alpha=(j \mid i)$ and $\beta=(l \mid k)$ in both these subsystems. One thus requires the triply-conditional probability $P[(j \mid i) \mid(l \mid k)]$, of the variable (conditional) two-orbital event (in one fragment, of the $i \rightarrow j$ scattering, conditional on the parameter (conditional) two-orbital event in another fragment, of the $k \rightarrow l$ probability propagation. Clearly, the AO labels in each subspace and in both conditional events may be repeated, to account for the intra-subsystem bond contributions.

Earlier attempts [1,5] to solve a seemingly non-unique problem of generating such propagation-coupling, triply-conditional probabilities $P[(i \mid j) \mid(k \mid l)]$ and the associated joint probabilities $P[(i \mid j) \wedge(k \mid l)]=P[(i \mid j) \mid(k \mid l)] P(k \mid l) \equiv P[(i \mid j),(k \mid l)]$, was shown to violate the requirements of the non-negativity and of relevant symmetries with respect to exchange of orbitals, although for equal $\mathrm{AO}$ occupations they satisfy Bayes' rule,

$$
P(\beta \mid \alpha)=P(\beta) P(\alpha \mid \beta) / P(\alpha)=P(\beta) P(\alpha \mid \beta) /\left[\sum_{\beta} P(\beta) P(\alpha \mid \beta)\right],
$$

about "hypotheses" $\{\beta\}$ accounting for the occurrence of $\alpha$. Its first part implies that the probability ratio of individual propagations can be expressed as the corresponding ratio of their reverse conditional probabilities:

$$
P(\beta) / P(\alpha)=P(\beta \mid \alpha) / P(\alpha \mid \beta) .
$$

The underlying joint probabilities of the simultaneous four-orbital events in the molecular bond space, $P[(i \wedge j) \wedge(k \wedge l)] \equiv P(i, j, k, l)$, originate from the AO participation in the system ground-state. As we have already recognized above, the conditioning of $\chi_{j}$ on $\chi_{i}$ amounts to the projection upon the variable orbital $\chi_{j}$ on the reference orbital $\chi_{i}$ in the bonding subspace of MO. This operation is effected by the scattering operator $\hat{\mathrm{S}}_{i}$. The subspace generalization of this procedure, involving a trace over one bond-subspace of the projection onto another bond-subspace, similarly generates the singly-conditional probability $P\left(\varepsilon_{1} \mid \varepsilon_{2}\right)$. The triply-conditional probabilities $P[(j \mid i) \mid(l \mid k)]$ can thus be viewed as being derived from a sequence of such three conditioning operations $\hat{\mathrm{C}}(\zeta \mid \xi)$ performed on molecular basis functions, where $\zeta$ and $\xi$ denote either a single AO's or their pairs $\varepsilon_{1}=(i, j)$ and $\varepsilon_{2}=(k, l)$ : two operations $\hat{\mathrm{C}}(j \mid i)$ and $\hat{\mathrm{C}}(l \mid k)$ conditioning a pair of orbitals in each set, and the subspace-conditiong $\hat{\mathrm{C}}\left(\varepsilon_{1} \mid \varepsilon_{2}\right)$. 
For example, one can view the probability $P[(j \mid i) \mid(l \mid k)]$ as resulting from $P\left(\varepsilon_{1} \mid \varepsilon_{2}\right)$ by the extra-conditioning performed within each of the two-AO subspaces: $\hat{\mathrm{C}}(j \mid i) \hat{\mathrm{C}}(l \mid k) \hat{\mathrm{C}}\left(\varepsilon_{1} \mid \varepsilon_{2}\right)$. Alternatively, one can start from two conditional events in each of these subspaces, with probabilities $P(j \mid i)$ and $P(l \mid k)$, respectively, with subsequent conditioning of the two subspaces themselves: $\hat{\mathrm{C}}\left(\varepsilon_{1} \mid \varepsilon_{2}\right) \hat{\mathrm{C}}(j \mid i) \hat{\mathrm{C}}(l \mid k)$. These operations do not commute, however, with each scheme leading to non-negative though different expressions for $P[(j \mid i) \mid(l \mid k)], P[(i \mid j),(k \mid l)]$ and $P(i, j, k, l)$. The resulting expressions also fail to satisfy the Bayes rule.

Consider a particular example of the first of these conditioning sequences. As we have already recognized in Eq. 6, the conditioning within a given pair of $\mathrm{AO}$ amounts to the projection upon the reference orbital in the bonding subspace of MO. This operation is effected by the scattering operator $\hat{\mathrm{S}}_{i}$ [Eq. 7]. The triply-conditional probabilities $P[(j \mid i) \mid(l \mid k)]$ can now be viewed as derived from the subspace probability $P\left(\varepsilon_{1} \mid \varepsilon_{2}\right)$ [Eq. 17], where such extra-conditioning has been performed within each of the two-AO subspaces, since now both events to be conditioned are already conditional themselves. However, since the reference orbital $\chi_{i}$ in $[(j \mid i) \mid(l \mid k)]$ belongs to the variable subspace $\varepsilon_{1}$ the trace of Eq. 17 should now be performed over the $\hat{\mathrm{S}}_{i}$ projected subspace

$$
\varepsilon_{1}^{(i)} \equiv \hat{\mathrm{S}}_{i} \varepsilon_{1}=\left(\hat{\mathrm{S}}_{i}|i\rangle, \hat{\mathrm{S}}_{i}|j\rangle\right)=|\varphi\rangle\langle\varphi \mid i\rangle\left(\gamma_{i, i}, \gamma_{i, j}\right) \equiv\left|i^{b}\right\rangle\left(\gamma_{i, i}, \gamma_{i, j}\right)
$$

Similarly, since the other reference orbital $\chi_{k}$ in $[(j \mid i) \mid(l \mid k)]$ is a member of the reference subspace $\varepsilon_{2}$ the projection $\hat{\mathrm{S}}_{k}$ should now be performed on the operator part of Eq. 17:

$$
\hat{\mathrm{S}}_{\varepsilon_{2}}^{(k)} \equiv \hat{\mathrm{S}}_{k} \hat{\mathrm{S}}_{\varepsilon_{2}} \hat{\mathrm{S}}_{k}=\hat{\mathrm{P}}_{\varphi} \hat{\mathrm{P}}_{k} \hat{\mathrm{P}}_{\varphi}\left(\hat{\mathrm{P}}_{k}+\hat{\mathrm{P}}_{l}\right) \hat{\mathrm{P}}_{\varphi} \hat{\mathrm{P}}_{k} \hat{\mathrm{P}}_{\varphi}
$$

This procedure finally gives:

$$
\begin{aligned}
P[(j \mid i) \mid(l \mid k)] & =\mathcal{\gamma} \operatorname{Tr}_{\varepsilon_{1}(i)} \hat{\mathrm{S}}_{\varepsilon_{2}}^{(k)} \\
& =\mathcal{\alpha}\left(\gamma_{i, k}\right)^{2}\left[\left(\gamma_{i, i}\right)^{2}+\left(\gamma_{i, j}\right)^{2}\right]\left[\left(\gamma_{k, k}\right)^{2}+\left(\gamma_{k, l}\right)^{2}\right] \geq 0,
\end{aligned}
$$

where $\mathcal{\gamma}$ stands for the appropriate normalization constant.

It also follows from Eq. 23 that the projected subspace $\varepsilon_{1}^{(i)}$ contains two pieces of the bond-projection $\left|i^{b}\right\rangle=|\varphi\rangle\langle\varphi \mid i\rangle$ of orbital $\chi_{i}$, which determines the orbital electron occupation $\gamma_{i, i}=2\left\langle i^{b} \mid i^{b}\right\rangle$. Together they give rise to the overall norm

$$
\mathcal{\alpha}\left[\varepsilon_{1}^{(i)}\right]=1 / 2 \gamma_{i, i}\left[\left(\gamma_{i, i}\right)^{2}+\left(\gamma_{i, j}\right)^{2}\right] \text {, }
$$

given by the the trace of the subspace metric tensor:

$$
\left\langle\varepsilon_{1}^{(i)} \mid \varepsilon_{1}^{(i)}\right\rangle=\left\langle i^{b} \mid i^{b}\right\rangle\left[\begin{array}{ll}
\gamma_{i, i}^{2} & \gamma_{i, i} \gamma_{i, j} \\
\gamma_{i, j} \gamma_{i, i} & \gamma_{i, j}^{2}
\end{array}\right]=\frac{\gamma_{i, i}}{2}\left[\begin{array}{ll}
\gamma_{i, i}^{2} & \gamma_{i, i} \gamma_{i, j} \\
\gamma_{i, j} \gamma_{i, i} & \gamma_{i, j}^{2}
\end{array}\right] .
$$


When the two conditional events are interchanged, the associated norm of $\varepsilon_{2}^{(k)}$,

$$
\mathcal{\alpha}\left[\varepsilon_{2}^{(k)}\right]=1 / 2 \gamma_{k, k}\left[\left(\gamma_{k, k}\right)^{2}+\left(\gamma_{k, l}\right)^{2}\right]
$$

measures the trace of the associated bond-projected metric tensor

$$
\left\langle\varepsilon_{2}^{(k)} \mid \varepsilon_{2}^{(k)}\right\rangle=\left\langle k^{b} \mid k^{b}\right\rangle\left[\begin{array}{ll}
\gamma_{k, k}^{2} & \gamma_{k, k} \gamma_{k, l} \\
\gamma_{k, l} \gamma_{k, k} & \gamma_{k, l}^{2}
\end{array}\right]=\frac{\gamma_{k, k}}{2}\left[\begin{array}{ll}
\gamma_{k, k}^{2} & \gamma_{k, k} \gamma_{k, l} \\
\gamma_{k, l} \gamma_{k, k} & \gamma_{k, l}^{2}
\end{array}\right] .
$$

Therefore, it follows from Eqs. 35 and 37 that the triply-conditional probability of Eq. 34 is proportional to the above norms of the conditional subspaces:

$$
P[(j \mid i) \mid(l \mid k)]=\left\{4 \propto \gamma\left(\gamma_{i, k}\right)^{2} /\left(\gamma_{i, i} \gamma_{k, k}\right)\right\} \propto \gamma\left[\varepsilon_{1}^{(i)}\right] \propto \gamma\left[\varepsilon_{2}^{(k)}\right] .
$$

Finally, one can fix the normalization of such triply-conditional probability measures by the admissible requirement:

$$
P[(j \mid i) \mid(j \mid i)]=P(j \mid i) .
$$

In the spirit of Eq. 8 it relates this pair-diagonal, triply-conditional probability, of the (conditional) two-AO event conditional upon itself, to the probability of the conditional event itself. This equation gives the normalization constant,

$$
\gamma=\gamma_{i, j}^{2} /\left[2 \gamma_{i, i}^{3}\left(\gamma_{i, i}^{2}+\gamma_{i, j}^{2}\right)^{2}\right]
$$

and hence the final expression for the triply-conditional probability:

$$
P[(j \mid i) \mid(l \mid k)]=P(\alpha \mid \beta)=\frac{\gamma_{i, j}^{2} \gamma_{i, k}^{2}\left(\gamma_{k, k}^{2}+\gamma_{k, l}^{2}\right)}{2 \gamma_{i, i}^{3}\left(\gamma_{i, i}^{2}+\gamma_{i, j}^{2}\right)} \geq 0
$$

This form clearly distinguishes between the variable $(j \mid i)$ and parameter $(l \mid k)$ two orbital events. It also implies that a nonvanishing probability for the occupied AO, $\gamma_{i, i}>0$, requires the finite coupling between the reference orbitals of two subspaces, through $\gamma_{i, k} \neq 0$, and the chemical interaction between the two orbitals in the variable subspace: $\gamma_{i, j} \neq 0$. This accords with the intuitive expectation that conditioning of the electron scattering events (chemical bonds) on molecular fragments requires the chemical interactions between these subsystems.

It should be observed however, that the associated measure of the joint probability of the simultaneous occurrence of the two conditional events:

$$
P[(j \mid i),(l \mid k)]=P(l \mid k) P[(j \mid i) \mid(l \mid k)]=\frac{\gamma_{i, j}^{4} \gamma_{i, k}^{2}\left(\gamma_{k, k}^{2}+\gamma_{k, l}^{2}\right)}{4 \gamma_{i, i}^{4}\left(\gamma_{i, i}^{2}+\gamma_{i, j}^{2}\right)} \geq 0,
$$


still fails to satisfy the symmetry requirement with respect to exchange $\alpha \equiv(j \mid i) \leftrightarrow$ $(l \mid k) \equiv \beta$.

In designing the fully symmetrical measure of these triply conditional probabilities of $\mathrm{AO}$ events in the molecular bond system one can also follow the heuristic approach of Eqs. 25-27:

$$
P(\alpha \mid \beta)=\gamma_{\alpha, \beta}^{2} /\left(2 \gamma_{\beta, \beta}\right)
$$

where $\gamma_{\alpha, \beta}$ denotes the appropriate average chemical interaction attributed to two conditional AO events $\alpha$ and $\beta$, and $\gamma_{\beta, \beta}$ stands for the effective electron occupation associated with the reference conditional event. In order to determine these quantities we use the known probabilities of the separate events $\alpha$ and $\beta$, applied as weighting factors,

$$
\gamma_{\alpha, \beta}^{2}=P(\alpha) \gamma_{\varepsilon_{1}, \varepsilon_{2}}^{2} P(\beta), \quad \gamma_{\beta, \beta}=P(\beta) \gamma_{\varepsilon_{2}, \varepsilon_{2}}
$$

The emerging heuristic expression for the triply conditional probabilities then reads:

$$
\begin{aligned}
P(\alpha \mid \beta) & =P(\alpha) \gamma_{\varepsilon_{1}, \varepsilon_{2}}^{2} /\left(2 \gamma_{\varepsilon_{2}, \varepsilon_{2}}\right) \\
& =\left\{\left(\gamma_{i, j}\right)^{2}\left[4 \gamma_{i, i}\left(\gamma_{k, k}+\gamma_{l, l}\right)\right]^{-1}\right\}\left[\left(\gamma_{i, k}\right)^{2}+\left(\gamma_{i, l}\right)^{2}+\left(\gamma_{j, k}\right)^{2}+\left(\gamma_{j, l}\right)^{2}\right]
\end{aligned}
$$

The crucial test of its adequacy comes from calculating Bayes' ratio of Eq. 31:

$$
P(\beta \mid \alpha) / P(\alpha \mid \beta)=\left(\gamma_{k, l}\right)^{2} \gamma_{i, i}\left(\gamma_{k, k}+\gamma_{l, l}\right) /\left[\left(\gamma_{i, j}\right)^{2} \gamma_{k, k}\left(\gamma_{i, i}+\gamma_{j, j}\right)\right]
$$

to be compared with the probability ratio of individual conditional events:

$$
P(\beta) / P(\alpha)=\left(\gamma_{k, l}\right)^{2} \gamma_{i, i} /\left[\left(\gamma_{i, j}\right)^{2} \gamma_{k, k}\right]
$$

One observes that the symmetrical expression of Eq. 46 indeed satisfies Bayes' rule for equal occupations of the two orbital subspaces:

$$
N_{\varepsilon_{1}}=\gamma_{i, i}+\gamma_{j, j}=2 \gamma_{\varepsilon_{1}, \varepsilon_{1}}=N_{\varepsilon_{2}}=\gamma_{k, k}+\gamma_{l, l}=2 \gamma_{\varepsilon_{2}, \varepsilon_{2}}
$$

We can thus conclude that this probability can serve as another adequate measure of the propagation-coupling probabilities which emerge in the bond-coupling phenomena of OCT.

The additional test comes from the symmetry requirement of the resultant joint probabilities of the two conditional events:

$$
P(\alpha \wedge \beta)=P(\beta) P(\alpha \mid \beta)=P(\alpha) P(\beta \mid \alpha) .
$$


The two probability products respectively give:

$$
\begin{aligned}
& P(\beta) P(\alpha \mid \beta)=P(\alpha) \gamma_{\varepsilon_{1}, \varepsilon_{2}}^{2} P(\beta) /\left(2 \gamma_{\varepsilon_{2}, \varepsilon_{2}}\right)=\gamma_{\alpha, \beta}^{2} / N_{\varepsilon_{2}} \quad \text { and } \\
& P(\alpha) P(\beta \mid \alpha)=P(\alpha) \gamma_{\varepsilon_{1}, \varepsilon_{2}}^{2} P(\beta) /\left(2 \gamma_{\varepsilon_{1}, \varepsilon_{1}}\right)=\gamma_{\alpha, \beta}^{2} / N_{\varepsilon_{1}} .
\end{aligned}
$$

These probabilities are indeed equal for equal occupations of the two orbital subspaces. In practical molecular calculations the average of these two estimates should be used as the fully symmetrical joint-probability measure:

$$
\begin{aligned}
P(\alpha \wedge \beta) & =1 / 2[P(\beta) P(\alpha \mid \beta)+P(\alpha) P(\beta \mid \alpha)] \\
& =1 / 2 \gamma_{\alpha, \beta}^{2}\left[1 / N_{\varepsilon_{1}}+1 / N_{\varepsilon_{2}}\right] \equiv \frac{\gamma_{\alpha, \beta}^{2}}{2 N_{\left(\varepsilon_{1}, \varepsilon_{2}\right)}^{h}},
\end{aligned}
$$

which assumes a general form of the two-orbital expression of Eq. 10 when expressed in terms of the harmonic average $N_{\left(\varepsilon_{1}, \varepsilon_{2}\right)}^{h}$ of the two subspace occupations.

This expression approximately satisfies the associated normalization requirements for joint probabilities,

$$
\begin{aligned}
\sum_{\alpha} P(\alpha \wedge \beta) & =\left[2 N_{\left(\varepsilon_{1}, \varepsilon_{2}\right)}^{h}\right]^{-1} \sum_{\alpha} \gamma_{\alpha, \beta}^{2} \\
& =\left[2 N_{\left(\varepsilon_{1}, \varepsilon_{2}\right)}^{h}\right]^{-1}\left[\sum_{\alpha} P(\alpha)\right]\left[\operatorname{tr}_{\left\{\varepsilon_{1}\right\}} \gamma_{\varepsilon_{1}, \varepsilon_{2}}^{2}\right] P(\beta) \\
& =\left[N_{\varepsilon_{2}} / 2 N_{\left(\varepsilon_{1}, \varepsilon_{2}\right)}^{h}\right] P(\beta) \approx P(\beta)
\end{aligned}
$$

for equal electron occupations of the two subspaces, when $N_{\varepsilon_{2}}=2 N_{\left(\varepsilon_{1}, \varepsilon_{2}\right)}^{h}$.

\section{Illustrative application to $\pi$-electron systems}

The preliminary approach to multi-conditional events of $\pi$-electrons in butadiene and benzene have recently been discussed using the Hückel CBO data [5]. Since the bondprojected, coupling measure of the many-AO "probabilities" adopted in this study exhibited several shortcomings, it is of interest to reexamine this interesting chemical problem using the present multi-bond probabilities generated by Eqs. 42 and 47, derived from the bond-projected superposition principle for subspaces of basis functions, which are free of some of these fundamental deficiencies. In what follows we shall apply the ground-state density matrices reported in ref. [5] for butadiene and benzene, for the consecutive numbering of the $2 p_{\pi}$ orbitals contributed by the constituent carbon atoms.

Let us first examine predictions from Eq. 42. For butadiene the present approach gives the following (symmetry-unrelated) pair-diagonal probabilities involving different $\mathrm{AO}$ : 


$$
\begin{aligned}
& P[(1 \mid 2) \mid(1 \mid 2)]=P(1 \mid 2)=0.40, \\
& P[(1 \mid 4) \mid(1 \mid 4)]=P(1 \mid 4)=P[(2 \mid 3) \mid(2 \mid 3)]=P(2 \mid 3)=0.10, \\
& P[(1 \mid 3) \mid(1 \mid 3)]=P(1 \mid 3)=0.00 .
\end{aligned}
$$

They reflect differences between the strong information couplings of the probability scatterings in the peripheral $1-2(3-4) \pi$ bonds, a weaker chemical interaction inside the middle $2-3$ bond and the 1-4 "bond" connecting peripheral atoms, and the absence of any interaction between "bonds" connecting the chemically non-bonded pairs of atoms: 1-3 (2-4).

Using Eq. 42 one similarly finds the representative probabilities for the pairnon-diagonal events in the $\pi$-electron system of butadiene:

$$
\begin{aligned}
& P[(1 \mid 1) \mid(2 \mid 1)]=0.45, \quad P[(1 \mid 1) \mid(4 \mid 1)]=0.30, \quad P[(1 \mid 1) \mid(3 \mid 1)]=0.25, \\
& P[(1 \mid 2) \mid(3 \mid 2)]=0.27, \quad P[(1 \mid 2) \mid(4 \mid 1)]=P[(1 \mid 2) \mid(2 \mid 3)]=0.21, \\
& P[(1 \mid 2) \mid(3 \mid 1)]=0.18, \quad P[(1 \mid 4) \mid(2 \mid 3)]=P[(1 \mid 2) \mid(4 \mid 3)]=0.08, \\
& P[(1 \mid 2) \mid(1 \mid 3)]=0.04, \\
& P[(1 \mid 2) \mid(1 \mid 4)]=P[(1 \mid 2) \mid(3 \mid 4)]=P[(1 \mid 3) \mid(3 \mid 4)]=0.00 .
\end{aligned}
$$

The vanishing non-diagonal probabilities, in the last row of the preceding equation, are due to the absence of the chemical interaction between the two reference orbitals $\left(\gamma_{i, k}=0\right)$ and/or in the variable subspace $\left(\gamma_{i, j}=0\right)$ in the symbol of the triple-conditional event. The preceding, non-vanishing probabilities correctly reflect the expected strength of the inter-bond probability scatterings involving neighboring and more distant atoms, the peripheral and middle bonds, and they correctly reflect a lesser weight of the probability scattering between non-bonded atoms.

The corresponding pair-diagonal probabilities for benzene reflect the strength of chemical $\pi$ bonds in the regular-hexagon ring of carbon atoms:

$$
\begin{aligned}
& P[(i \mid i) \mid(i \mid i)]=P(i \mid i)=0.50, \quad P[(i+1 \mid i) \mid(i+1 \mid i)]=P(i+1 \mid i)=0.22 \\
& P[(i+2 \mid i) \mid(i+2 \mid i)]=P(i+2 \mid i)=0.00 \\
& P[(i+3 \mid i) \mid(i+3 \mid i)]=P(i+3 \mid i)=0.06
\end{aligned}
$$

The representative pair-non-diagonal probabilities coupling two different $\pi$ bonds in the benzene ring read:

$$
\begin{aligned}
& P[(i \mid i) \mid(i+1 \mid i)]=0.36, \quad P[(i \mid i) \mid(i+2 \mid i)]=0.25 \\
& P[(i \mid i) \mid(i+3 \mid i)]=0.28, \quad P[(i \mid i) \mid(i+2 \mid i+1)]=0.16, \\
& P[(i \mid i) \mid(i+3 \mid i+2)]=P[(i+1 \mid i) \mid(i+3 \mid i+2)]=0.00, \\
& P[(i+1 \mid i) \mid(i+2 \mid i+1)]=0.10, \quad P[(i+1 \mid i) \mid(i+4 \mid i+3)]=0.02 .
\end{aligned}
$$

Again, these probabilities adequately reflect both the spatial separation of both the two atoms in each pair and the pair-separation in the benzene ring, as well as the chem- 
ical interactions between the constituent carbon atoms quantified by the respective bond-orders.

Next, let us summarize the corresponding propagation-coupling predictions from the most symmetrical Eq. 47, which was shown to be heuristically related to the singly/conditional probabilities and to satisfy Bayes's hypotheses relations. For butadiene one obtains the following triply/conditional probabilities:

$$
\begin{aligned}
& P[(1 \mid 2) \mid(1 \mid 2)]=0.36, \quad P[(1 \mid 4) \mid(1 \mid 4)]=P[(2 \mid 3) \mid(2 \mid 3)]=0.06, \\
& P[(1 \mid 3) \mid(1 \mid 3)]=0.00 ;
\end{aligned}
$$

$$
\begin{aligned}
& P[(1 \mid 1) \mid(2 \mid 1)]=0.45, \quad P[(1 \mid 1) \mid(4 \mid 1)]=0.30, \quad P[(1 \mid 1) \mid(3 \mid 1)]=0.25, \\
& P[(1 \mid 2) \mid(3 \mid 2)]=P[(1 \mid 2) \mid(4 \mid 1)]=P[(1 \mid 2) \mid(2 \mid 3)]=P[(1 \mid 2) \mid(3 \mid 1)]=0.20, \\
& P[(1 \mid 4) \mid(2 \mid 3)]=P[(1 \mid 2) \mid(4 \mid 3)]=0.04, \quad P[(1 \mid 2) \mid(3 \mid 4)]=0.04, \\
& P[(1 \mid 2) \mid(1 \mid 3)]=P[(1 \mid 2) \mid(1 \mid 4)]=0.20, \quad P[(1 \mid 3) \mid(3 \mid 4)]=0.00 .
\end{aligned}
$$

The corresponding heuristic probabilities for benzene read:

$$
\begin{aligned}
& P[(i \mid i) \mid(i \mid i)]=0.50, \quad P[(i+1 \mid i) \mid(i+1 \mid i)]=P(i+1 \mid i)=0.16, \\
& P[(i+2 \mid i) \mid(i+2 \mid i)]=0.00, \quad P[(i+3 \mid i) \mid(i+3 \mid i)]=P(i+3 \mid i)=0.03
\end{aligned}
$$

$$
\begin{aligned}
& P[(i \mid i) \mid(i+1 \mid i)]=0.36, \quad P[(i \mid i) \mid(i+2 \mid i)]=0.25, \quad P[(i \mid i) \mid(i+3 \mid i)]=0.28, \\
& P[(i \mid i) \mid(i+2 \mid i+1)]=0.11, \quad P[(i \mid i) \mid(i+3 \mid i+2)]=0.03, \\
& P[(i+1 \mid i) \mid(i+3 \mid i+2)]=0.09, \\
& P[(i+1 \mid i) \mid(i+2 \mid i+1)]=0.10, \quad P[(i+1 \mid i) \mid(i+4 \mid i+3)]=0.01 . \quad(57 \mathrm{~b})
\end{aligned}
$$

Open Access This article is distributed under the terms of the Creative Commons Attribution Noncommercial License which permits any noncommercial use, distribution, and reproduction in any medium, provided the original author(s) and source are credited.

\section{References}

1. R.F. Nalewajski, Information Origins of the Chemical Bond (Nova, NewYork, 2010)

2. R.F. Nalewajski, Int. J. Quantum Chem. 109, 425 (2009)

3. R.F. Nalewajski, J. Math. Chem. 47, 692 (2010)

4. R.F. Nalewajski, J. Math. Chem. 47, 709 (2010)

5. R.F. Nalewajski, J. Math. Chem. 47, 808 (2010)

6. R.F. Nalewajski, Information Theory of Molecular Systems (Elsevier, Amsterdam, 2006), and refs. therein

7. C.E. Shannon, Bell Syst. Tech. J. 27, 379, 623 (1948)

8. N. Abramson, Information Theory and Coding (McGraw-Hill, New York, 1963)

9. P.E. Pfeifer, Concepts of Probability Theory, 2nd edn. (Dover, New York, 1978)

10. P.A.M. Dirac, The Principles of Quantum Mechanics, 4th edn. (Clarendon, Oxford, 1958) 
11. K.A. Wiberg, Tetrahedron 24, 1083 (1968)

12. M.S. Gopinathan, K. Jug, Theoret. Chim. Acta (Berl.) 63, 497, 511 (1983)

13. K. Jug, M.S. Gopinathan, in Theoretical Models of Chemical Bonding, vol. 2, ed. by Z.B. Maksić (Springer, Heidelberg, 1990), p. 77

14. I. Mayer, Chem. Phys. Lett. 97, 270 (1983)

15. R.F. Nalewajski, A.M. Köster, K. Jug, Theoret. Chim. Acta (Berl.) 85, 463 (1993)

16. R.F. Nalewajski, J. Mrozek, Int. J. Quantum Chem. 51, 187 (1994)

17. R.F. Nalewajski, S.J. Formosinho, A.J.C. Varandas, J. Mrozek, Int. J. Quantum Chem. 52, 1153 (1994)

18. R.F. Nalewajski, J. Mrozek, G. Mazur, Can. J. Chem. 100, 1121 (1996)

19. R.F. Nalewajski, J. Mrozek, A. Michalak, Int. J. Quantum Chem. 61, 589 (1997)

20. J. Mrozek, R.F. Nalewajski, A. Michalak, Polish J. Chem. 72, 1779 (1998)

21. R.F. Nalewajski, Chem. Phys. Lett. 386, 265 (2004) 